\title{
ARE ELLIPTICAL GALAXIES REALLY METAL-RICH?
}

\author{
And, If Not, Then What?
}

\author{
M. LOEWENSTEIN AND R. F. MUSHOTZKY \\ $N A S A / G S F C$ \\ Code 662, Greenbelt, MD 20771, USA
}

\section{Introduction}

In elliptical galaxies, where most of the stars - and therefore most of the heavy elements - were formed at an early epoch, the total mass, spatial distribution, and relative abundances of metals are intimately connected to the galaxy formation process.

Metallicities of the stars in elliptical galaxies can be estimated from optical broad-band photometry and spectroscopic measurement of selected absorption line indices. However, the transformation from these indirect abundance indicators to true stellar metallicites is highly non-trivial, depending on a priori assumptions about stellar physics details and star formation history. Since the hot interstellar medium primarily originates from stellar mass loss, X-ray observations provide independent and complementary abundance information. Moreover, there are distinct advantages to using strong $\mathrm{X}$-ray emission features over weak optical absorption features: the former are related to actual abundances in a much more direct, modelindependent, manner; and, one can determine abundances much further out in radius. 30-40 early-type galaxies have been observed with $A S C A$, representing the first large sample of high quality, broad-band X-ray spectra of elliptical galaxies. Most of these have been analyzed in detail by $\mathrm{K}$. Matsushita, and we draw upon the results presented in her thesis, as well as our own analysis, in this review.

\section{Abundances in Gas-Rich Ellipticals}

$A S C A$ spectra can generally be decomposed into soft and hard components (Matsumoto et al. 1997). The soft component originates in the hot (0.3-1 $\mathrm{keV}$ ) ISM, and shows a wide range of X-ray-to-optical flux ratios and Xray extents for any given optical luminosity. The hard component generally 
scales linearly with optical luminosity, with a relative normalization and spectrum consistent with measurements of the integrated emission from low mass X-ray binaries in spiral galaxy bulges. Figure 1 (from Matsumoto et al. 1997) compares $A S C A$ Solid State Imaging Spectrometer (SIS) spectra of the elliptical galaxies NGC 4365 and NGC 4636 . Note that these galaxies have similar optical luminosities and hard components; however, the soft component in NGC 4636 is nearly two orders of magnitude more luminous than that in NGC 4365. Since abundance uncertainties become large as the hard component begins to dominate and the equivalent widths of emission lines are diluted (e.g., for NGC 4365), this review focuses on observations of eighteen gas-rich ellipticals (e.g., NGC 4636) in the $A S C A$ archive - galaxies with high X-ray-to-optical fluxes based on ROSAT PSPC observations and low hard component fractions based on $A S C A$ spectral decomposition. The $\mathrm{X}$-ray emission extends well beyond the optical isophotes for some of these systems, but is more compact in others.

The SISO spectra of NGC4636 and NGC4365

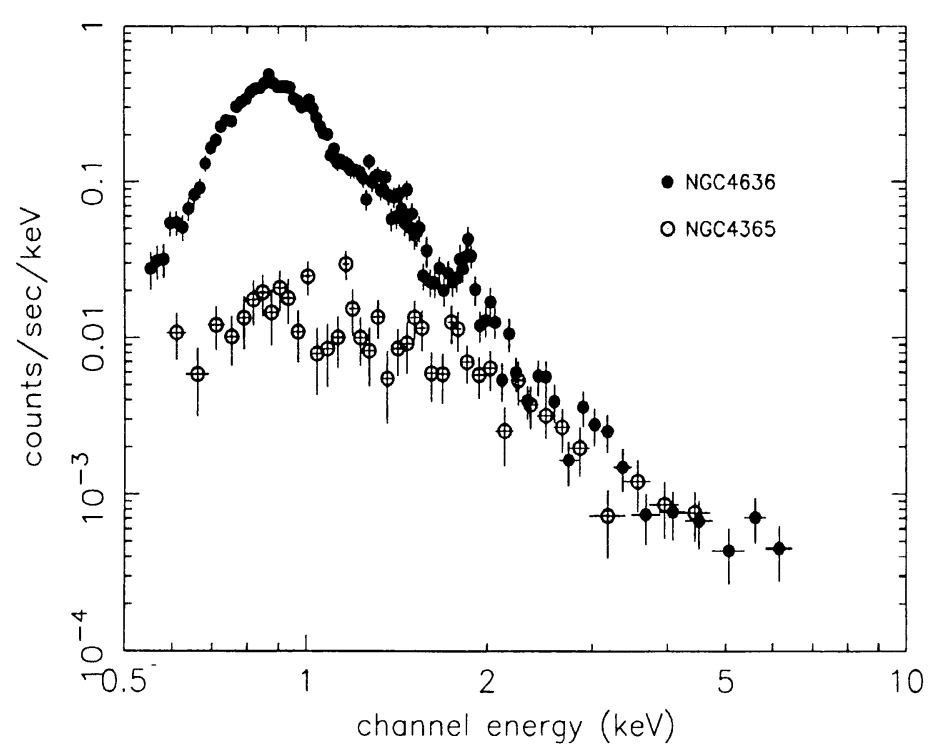

motumoto $30-0 c t-199622: 26$

Figure 1. Co-added ASCA SIS spectra of NGC 4636 (filled circles) and NGC 4365 (open circles).

Figure 2 shows a plot of abundance versus temperature derived from $A S C A$ spectra extracted from the inner five optical half-light radii. The soft component is modeled using the Raymond-Smith thermal plasma emission 
code with abundances fixed at their solar photospheric ratios. The abundances - essentially the Fe abundance, since X-ray spectra at these temperatures are dominated by $\mathrm{Fe} \mathrm{L}$ emission lines - range from about one-tenth to two-thirds solar. Since it has generally been assumed that abundances of the mass-losing stars that are the origin of the hot gas are supersolar, and that Type Ia SN should further enrich the hot gas by at least an additional two times solar, X-ray abundances of elliptical galaxies are 3-30 times lower than what might naively be expected.

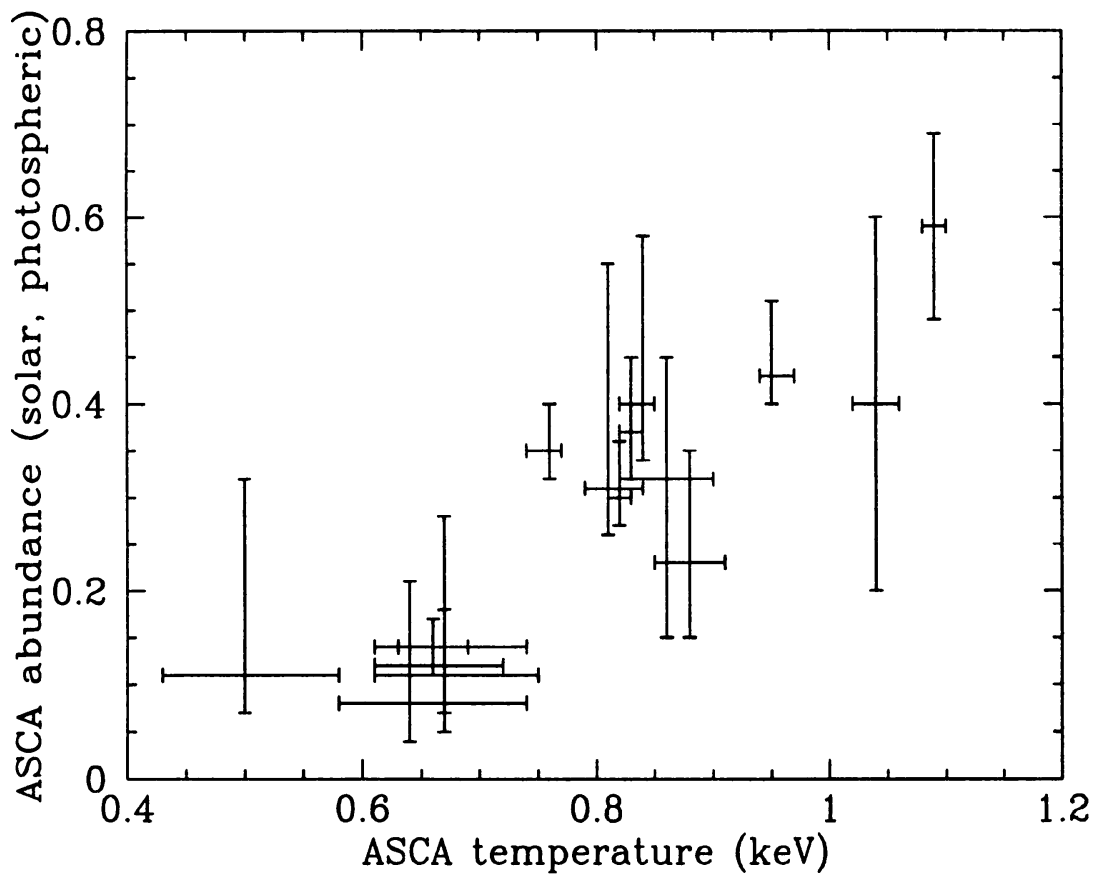

Figure 2. Hot gas metal abundance versus temperature.

\section{Reconciling Optical and X-ray Abundances}

Clearly, either the SNIa rate is much lower than estimated, and/or SNIa ejecta is not efficiently mixed into the hot ISM. Can optical and X-ray abundances be reconciled, even if SNIa enrichment is neglected? It must be the case that either (1) stellar abundances are not actually supersolar, (2) hot gas abundances are not actually subsolar, or (3) X-ray and optical observations are not commensurate. We consider these possibilities in reverse order. 
(3) We now know that the X-ray emission from some elliptical galaxies is much more extended than the optical light and, accounting for the measured abundance distributions, that the radius containing half of the hot gas metals is 3-10 times larger than the corresponding radius for the stars in such systems. The hot gas metallicity profile generally has a negative gradient (Matsushita 1997). These facts indicate that accretion of primordial or intergalactic material may be diluting the abundances of the hot gas relative to the stars (Brighenti \& Mathews 1997). However, if this were a dominant effect one would expect lower average metallicities in more gasrich galaxies - if anything, the opposite is observed. Although gas flows have undoubtedly rearranged and in some cases diluted the gas, it is probably meaningful to compare optical and X-ray metallicities within the optical radius.

(2) The validity of the abundances obtained from single-phase hot gas plus X-ray binaries fits to $A S C A$ data have been questioned on a number of fronts. Inadequacies in the treatment of $\mathrm{Fe} L$ transitions in plasma codes used for spectral fitting are an extra source of uncertainty (Arimoto et al. 1997); however, excluding the energy region in question in spectral fits does not systematically raise the metallicity (Buote \& Fabian 1997). Higher abundances can also be accommodated in more complex spectral models. In particular, Buote and Fabian have recently found that the best fit to $A S C A$ data often consists of a two-temperature plasma, with the secondary component having a temperature of $\sim 1.5 \mathrm{keV}$, and that the abundances in such fits are systematically higher by about a factor of two compared to models with a single gas phase plus X-ray binaries. However, there is more information in $A S C A$ spectra than what can be obtained from global spectral fits alone. We have found that the temperature obtained from the He-like to $\mathrm{H}$-like $\mathrm{Si}$ line ratios is in excellent agreement with the single-phase model (see Figure 3 for an example with a typical level of agreement), and is not consistent with the presence of hotter gas in the amounts suggested by Buote and Fabian.

(1) So, it seems reasonable to adopt the metallicities in the single-phase hot gas plus X-ray binaries fits to $A S C A$ spectra as measures of global interstellar Fe abundances, and sensible to compare these with global Fe abundances of the stars. What are the best current estimates of the latter? The conventional wisdom that elliptical galaxies have supersolar abundances is based on measurements of the nuclear Mg2 index. However, when one accounts for the factor of 2-3 overabundance of $\mathrm{Mg}$ with respect to $\mathrm{Fe}$ in the stars (Worthey et al. 1992), and the factor of two aperture correction (Arimoto et al. 1997) it becomes clear that the global Fe abundances of the stars and hot gas are not grossly discordant. We have compared recent estimates of the global $\mathrm{Fe}$ abundance using optical data kindly provided by S. Trager 


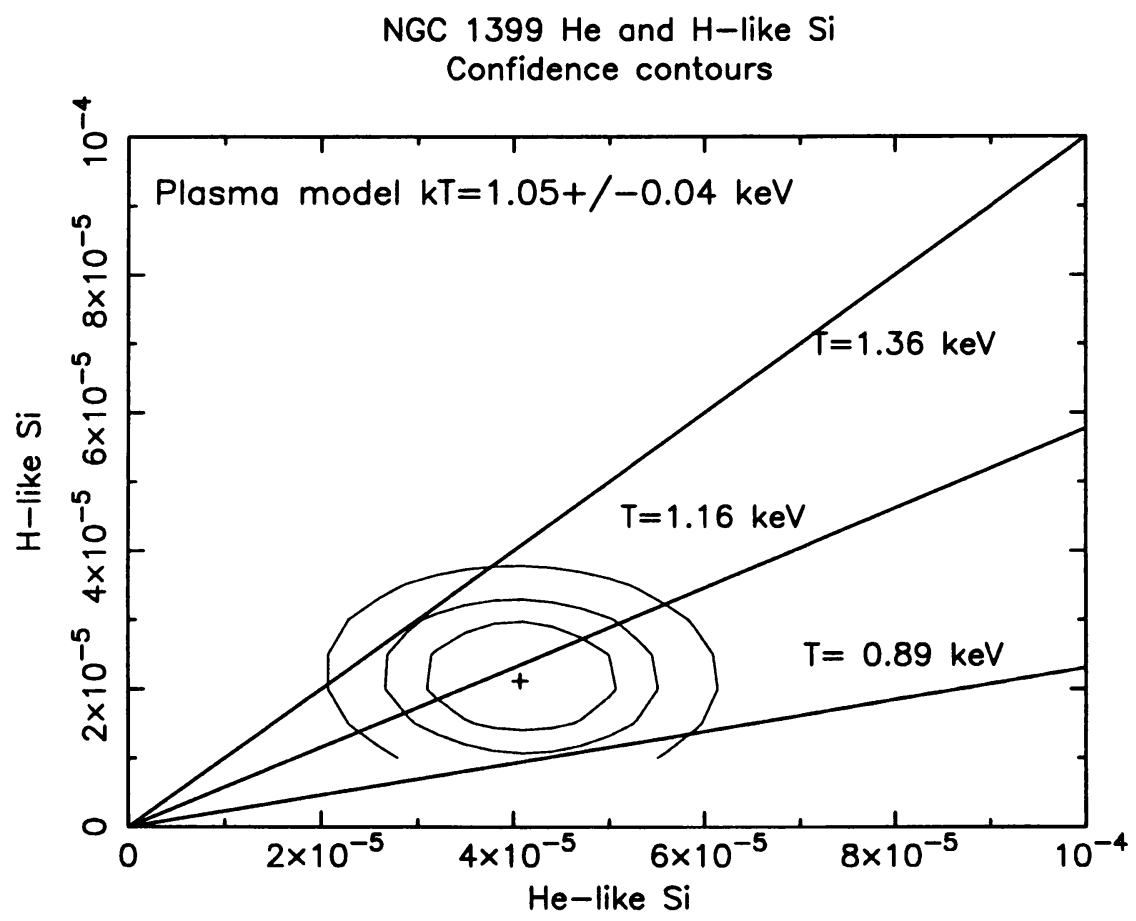

Figure 3. 68, 90, and 99\% confidence contours for $\mathrm{H}-$ and He-like Si line strengths (in photons $\mathrm{cm}^{-2} \mathrm{~s}^{-1}$ ) in the elliptical galaxy NGC 1399. The solid lines show the ratios expected in three single-temperature thermal plasma models. The best-fit temperature to the spectrum derived from global Raymond-Smith plasma model fits is $1.05 \pm 0.04$ $\mathrm{keV}$ ( $90 \%$ confidence uncertainties).

(Trager 1997) with the X-ray measurements. The comparison for the eight galaxies present in both samples is shown in Figure 4. The discrepancy is generally minor, although a few galaxies still have unaccountably low X-ray abundances. The average optical $\mathrm{Fe}$ abundances are about 0.45 solar (as predicted in the chemically consistent evolutionary models of Moller et al. 1997) compared to 0.3 solar for the hot gas.

\section{Relative Abundances}

Elemental abundance ratios provide constraints on the primordial IMF and relative numbers of Type Ia and Type II supernovae. Given sufficient signalto-noise, $\mathrm{O}, \mathrm{Mg}, \mathrm{Si}, \mathrm{S}$, and $\mathrm{Fe}$ all have prominent, distinct features in $A S C A$ spectra of elliptical galaxies. In variable abundance fits of seven ellipticals, we find Si-to-Fe ratios consistent with or perhaps somewhat less than the (meteoritic) solar value (Figure 5). This is lower than the Mg-to-Fe ratio derived from nuclear optical spectra, and is more in line with what has been 


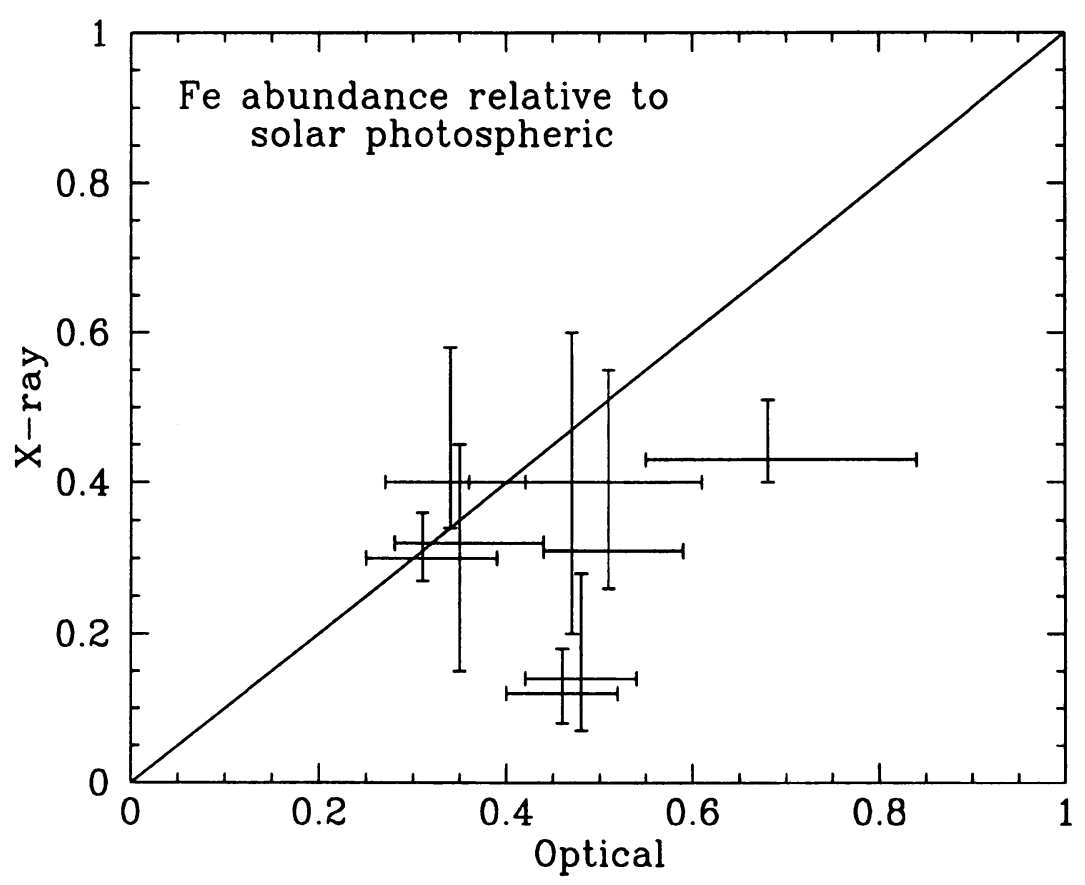

Figure 4. X-ray versus optical global iron abundance.

measured from the X-ray spectra of intergroup media. The $\mathrm{Si}$ abundance provides an independent and robust (plasma code uncertainties are very small for these $\mathrm{Si}$ lines) strong upper limit on the effective SNIa rate that is consistent with what is derived using Fe. The limit of about 0.03 SNU ( $1 \mathrm{SNU}=1$ supernova per century per $10^{10} L_{B \odot}$ ) is about four times lower than the recent estimate of Cappellaro et al. (1997) for $H_{o}=75 \mathrm{~km} \mathrm{~s}^{-1}$ $\mathrm{Mpc}^{-1}$

\section{Implications of Low Abundances for ICM Enrichment}

We have shown that the mass-averaged $\mathrm{Fe}$ abundance in an elliptical galaxy, based on X-ray and optical observations, is about one-half solar. This is only slightly higher than what is typically measured in the intracluster medium, and yet the mass in the ICM is generally $2-10$ times greater. There is several times more $\mathrm{Fe}$ (and $\mathrm{Si}$, as well) in the ICM than is locked up in stars in cluster galaxies. This implies the following.

(1) If the stellar and ICM metals come from the same SNII-enriched proto-elliptical galaxy gas, then $50-90 \%$ of the original galaxy mass has been lost and a significant fraction of the ICM is not primordial but has 


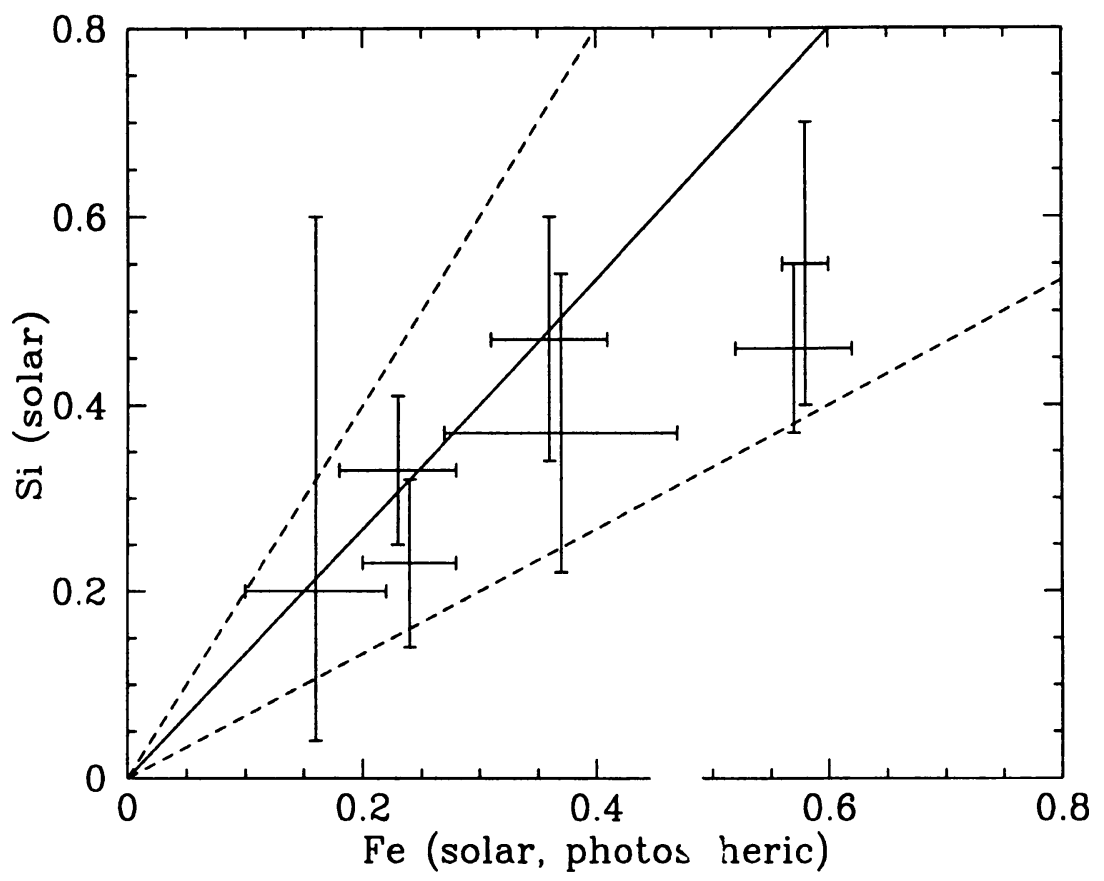

Figure 5. Si versus $\mathrm{Fe}$ abundance in the hot X-ray emitting gas. The solid line denotes $\mathrm{Si}: \mathrm{Fe}$ in the ratio $1: 1$, while the broken lines denote the ratios $3: 2$ and $1: 2$ with respect to the (meteoritic) solar ratio.

been ejected from galaxies. (2) However, the actual amount of material directly associated with the SNII ejecta is roughly an order of magnitude less. If there is selective mass-loss of nearly pure SNII ejecta it is possible to lose most of the metals without losing most of the mass.

(3) It is also possible that there is an additional significant source of ICM enrichment, although there are some difficulties with such a scenario (Gibson and Matteucci 1997). Perhaps, alternate sources of enrichment should be re-examined in light of the downward revision of elliptical galaxy abundances; although, one must bear in mind that ICM Fe mass is highly correlated with the total luminosity in elliptical galaxies (Arnaud et al. 1992).

\section{Concluding Remarks}

$\mathrm{X}$-ray spectra of elliptical galaxies are adequately fit by models consisting of hot gas with subsolar Fe abundance and roughly solar Si-to-Fe ratio, plus a hard component from an ensemble of X-ray binaries. The consistency of the relative magnitude and spectrum of the hard component with that 
expected from X-ray binaries, along with its more compact spatial distribution supports this model over ones where the hard component is primarily due to a hotter gas phase. Complications in the form of an extra soft continuum or multiple phases can be considered, but the consistency of the $\mathrm{Si}$ line diagnostic and continuum temperatures demonstrates that the data at the present level of sensitivity and spectral resolution - do not require these. Optical and X-ray Fe abundance estimates are converging, although there are some cases with anomalously low X-ray values. Problems in the Fe L spectral region remain; however, the main effect of improvements in atomic physics parameters is likely to be improved spectral fits rather than a radical upward revision in abundances.

Occam's razor would seem to demand that we provisionally accept the reality of low abundances in elliptical galaxies. As a result, we need to seriously reevaluate our notions of elliptical galaxy chemical evolution, intracluster enrichment, and Type Ia supernova rates.

We are grateful to Scott Trager and Kyoko Matsushita for making results from their dissertations available to us prior to publication.

\section{References}

Arimoto, N., Matsushita, K., Ishimaru, Y., Ohashi, T., \& Renzini, A. 1997, ApJ, 477, 128

Arnaud, M., Rothenflug, R., Boulade, O., Vigroux, L., \& Vangioni-Flan, E. 1992, $A \& A$, 254, 49

Brighenti, F. \& Mathews, W. G. 1997, $A p J$, in press

Buote, D. A., \& Fabian, A. C. 1997, MNRAS, submitted

Cappellaro, E., Turatto, M., Tsvetkov, D. Yu., Bartunov, O. S., Pollas, C., Evans, R., \& Hamuy, M. 1997, $A \mathcal{E} A, 322,431$

Gibson. B. K., \& Matteucci, F. 1997, ApJ, 475, 47

Matsumoto, H., Koyama, K., Awaki, H., Tsuru, T., Loewenstein, M., \& Matsushita, K. 1997, ApJ, 482, 133

Matsushita, K. 1997, Ph.D. thesis, University of Tokyo

Moller, C. S., Fritze-v. Alvensleben, U. \& Fricke, K. J. 1997, A\& A, 317, 676

Trager, S. C. 1997, Ph.D. thesis, University of California, Santa Cruz

Worthey, G., Faber, S. M., \& Gonzalez, J. J. 1992, ApJ, 398, 69 